\title{
Navigated iliac screw placement may reduce radiation and OR time in lumbopelvic fixation of unstable complex sacral fractures
}

\author{
M. F. Hoffmann ${ }^{1}$ - E. Yilmaz ${ }^{1}$ - D. C. Norvel ${ }^{2}$-T. A. Schildhauer ${ }^{1}$
}

Received: 3 November 2020 / Accepted: 26 January 2021 / Published online: 16 February 2021

(c) The Author(s) 2021

\begin{abstract}
Purpose Instability of the posterior pelvic ring may be stabilized by lumbopelvic fixation. The optimal osseous corridor for iliac screw placement from the posterior superior iliac spine to the anterior inferior iliac spine requires multiple ap- and lateral-views with additional obturator-outlet and -inlet views.

The purpose of this study was to determine if navigated iliac screw placement for lumbopelvic fixation influences surgical time, fluoroscopy time, radiation exposure, and complication rates.

Methods Bilateral lumbopelvic fixation was performed in 63 patients. Implants were inserted as previously described by Schildhauer. A passive optoelectronic navigation system with surface matching on L4 was utilized for navigated iliac screw placement. To compare groups, demographics were assessed. Operative time, fluoroscopic time, and radiation were delineated.

Results Conventional fluoroscopic imaging for lumbopelvic fixation was performed in 32 patients and 31 patients underwent the procedure with navigated iliac screw placement. No differences were found between the groups regarding demographics, comorbidities, or additional surgical procedures.

Utilization of navigation led to fluoroscopy time reduction of more than $50 \%$ (3.2 vs. 8.6 min.; $p<0.001$ ) resulting in reduced radiation (2004.5 vs. $\left.5130.8 \mathrm{~Gy}^{*} \mathrm{~cm}^{2} ; p<0.001\right)$. Operative time was reduced in the navigation group (176.7 vs. $227.4 \mathrm{~min}$; $p=0.002$ ) despite the necessity of additional surface referencing.

Conclusion For iliac screws, identifying the correct entry point and angle of implantation requires detailed anatomic knowledge and multiple radiographic views. In our study, additional navigation reduced operative time and fluoroscopy time resulting in a significant reduction of radiation exposure for patients and OR personnel.
\end{abstract}

Keywords Navigation · Radiation · Lumbopelvic fixation · Fluoroscopy

\section{Introduction}

In trauma patients, the sacrum is the keystone of the pelvic ring [1]. Despite multiple operative stabilization techniques including open or percutaneous iliosacral screw osteosynthesis, tension band transiliac plate osteosynthesis, transiliac bars, and local plate osteosynthesis, fixation of sacral fractures continues to be challenging due to complex local anatomy, unique biomechanical forces, and often poor bone

M. F. Hoffmann

martinfhoffmann@gmx.net

1 Berufsgenossenschaftliches Universitätsklinikum Bergmannsheil Bochum, Bürkle-de-la-Camp-Platz 1, 44789 Bochum, Germany

2 Spectrum Research, Inc., Tacoma, WA, USA quality [2,3]. Especially in severe comminuted sacral fractures and fractures with horizontal fracture lines (Fig. 1a, b) lumbopelvic fixation introduced by Trenz et al. [4] provides superior stability [5] and allows immediate weight-bearing while unloading the area of injury [6-8]. Pedicle screw placement is a common procedure and presents no relevant difficulties. Identifying the correct entry point for the iliac screws and the correct angle of implantation in all planes is crucial, requires detailed anatomic knowledge, and multiple radiographic views [9]. The optimal osseous corridor for iliac screw placement requires multiple posteroanterior (PA) and lateral views (LAT) with additional obturator outlet and obturator inlet views. Obtaining the correct views results in increased fluoroscopy time and radiation exposure for the patient and OR personnel. 


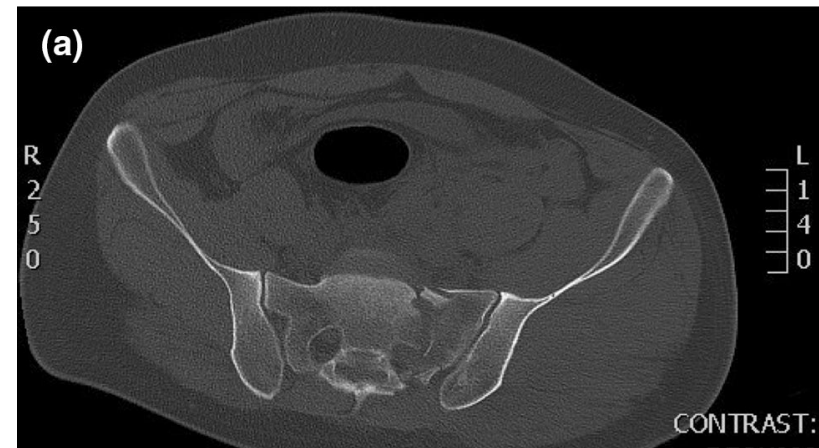

(b)

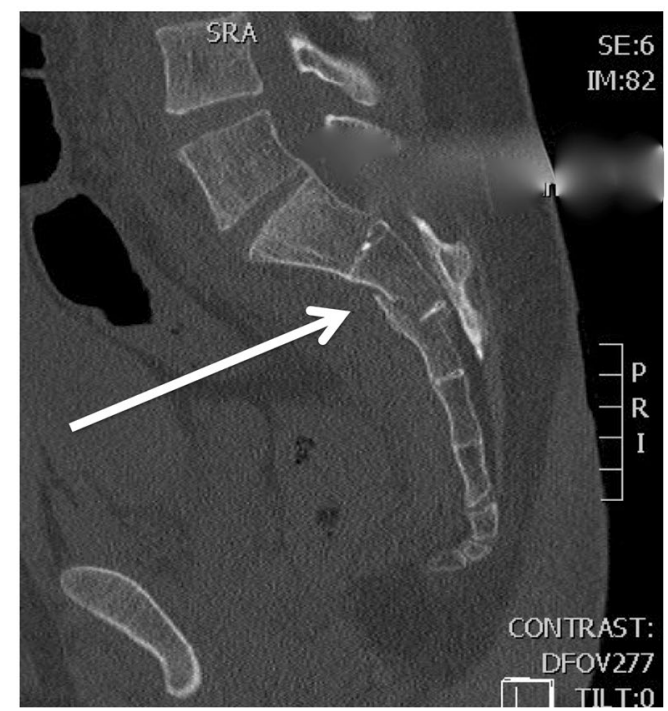

Fig. 1 CT scans of all patients have been performed. a Demonstrates a comminuted sacral fracture. The sagittal reconstruction (b) shows the horizontal fracture line leading to rotational instability

Different navigation systems based on fluoroscopy or CT scans are established in orthopedic surgery and routinely utilized for pedicle screw placement. Previous studies suggest that computer assisted and navigated surgery for screw placement in pelvic fractures is advantageous and may result in increased accuracy of screw placement, reduction of fluoroscopy time, OR time, and radiation exposure [8, 10-12].

Therefore, the purpose of this study was to determine if navigated iliac screw placement for lumbopelvic fixation influences (1) surgical time, (2) fluoroscopy time, (3) radiation exposure, (4) accuracy of screw placement, and (5) complication rates.

\section{Materials and methods}

This study was an Institutional Review Board approved retrospective cohort study of consecutive patients undergoing surgical treatment of unstable sacral fractures treated with lumbopelvic fixation in a single level I trauma center. During the study period, we identified 77 patients that had

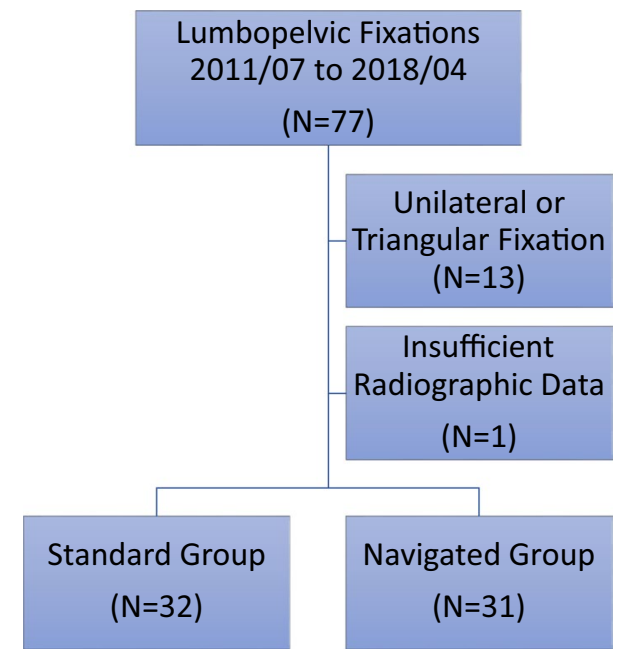

Fig. 2 Diagram illustrating the excluded patients and study groups

lumbopelvic fixation for pelvic fractures or instabilities from July 2011 through April 2018. Inclusion criteria were: bilateral surgical treatment for unstable sacral fractures or sacral instability and age equal to or older than 18 years. Exclusion criteria were insufficient medical and/or radiographic data, or triangular fixation (Fig. 2).

Each patient had three preoperative views of the pelvis. These were an anteroposterior (AP) view with the patient supine, a pelvic inlet view, and an outlet view. Inlet and outlet views were performed for assessing rotational, translational, and vertical displacement. Each patient had a CT scan with reconstruction of the injured pelvis that provided information on both extent of the injury and the magnitude of the displacement of the sacroiliac joint, the sacrum, or the iliac wing. Furthermore, the CT scan was utilized in the navigated group as the dataset for the $3 \mathrm{D}$ navigation and reconstruction.

Patients were positioned prone on a radiolucent table with appropriate eye protection and sequential compression devices. The entire posterior aspect of the pelvis and gluteal area was prepped and draped. A midline incision was performed down to the posterior elements. Attention to detail was maintained to avoid dural or neural element injury through the fracture sites. The operative approaches to the pelvis were tailored to each patient based on the particular pattern of the injury, location of the fracture, associated injuries, and soft tissue involvement [13]. Lumbopelvic implants (USS II, DePuySynthes, Paoli, PA) were inserted as described by Schildhauer through a midline incision [14]. The iliac screws were positioned in the bony canal between the posterior superior iliac spine (PSIS) and the anterior inferior iliac spine (AIIS), connected to pedicle screws by vertical rods and additionally stabilized by a transverse rod to prevent vertical shear. 
Patients were either operated in standard fashion utilizing intraoperative standard fluoroscopic views or underwent computer assisted and navigated surgery for iliac screw placement by the first author.

Standard iliac screw placement was performed in 32 patients utilizing anteroposterior (AP) and lateral views (LAT) with additional obturator outlet and obturator inlet views [9] (Fig. 3a, b). In 31 patients, a passive optoelectronic navigation system (Curve, Brainlab, Germany) was used for iliac screw placement (Fig. 4). Surface registration was performed on the lamina and spinous process of L4 for the matching procedure. Iliac screws were placed before any reduction maneuver. In both groups, no open reduction of the sacrum was required.

In all patients, a recession was created for the screw head, end cap, and connector rod in an attempt to reduce screw
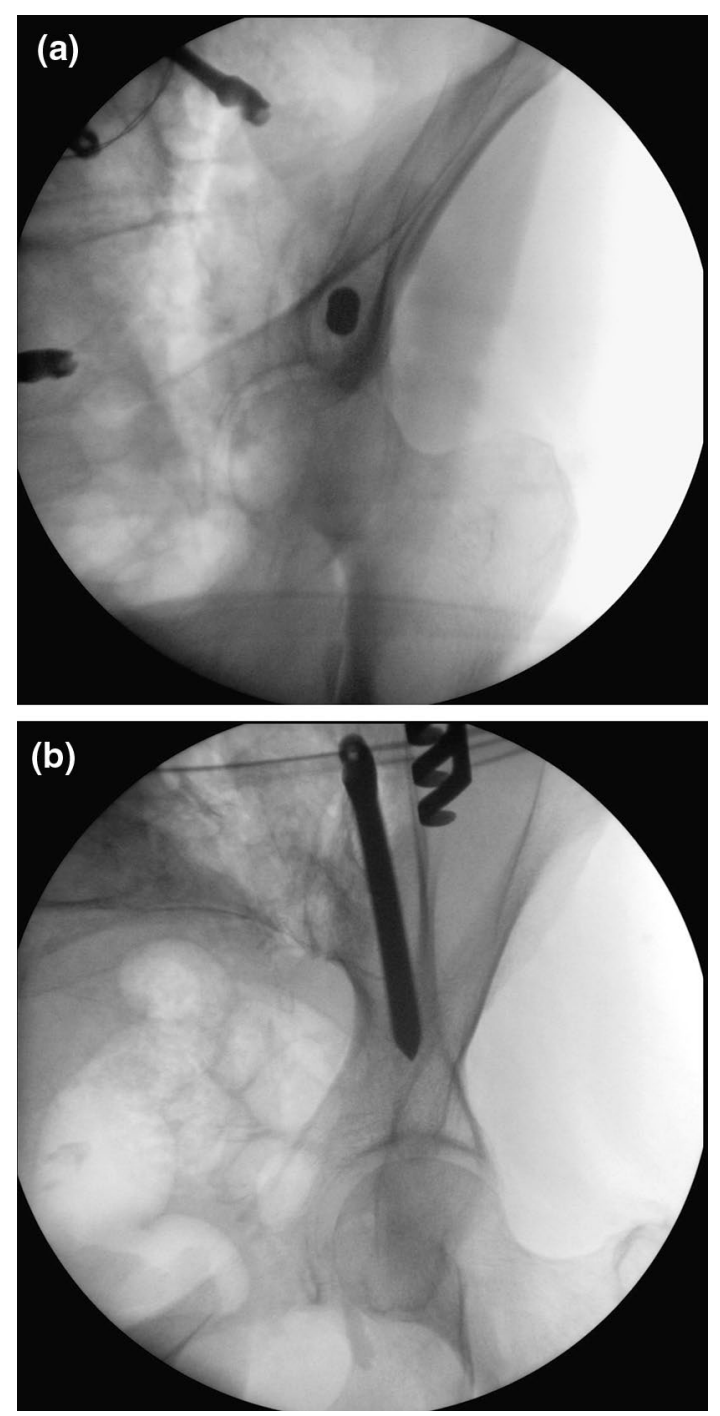

Fig. 3 a, b The intraoperative fluoroscopic views demonstrating the intraosseous placement of the iliac screws head prominence. Screw size was based upon length from the recessed entrance of the PSIS, along the sciatic buttress, and ending at the anterior inferior iliac spine (AIIS) and was 7.0 or $8.0 \mathrm{~mm} \times 110-130 \mathrm{~mm}$ (USS II, Titanium, DePuySynthes, Paoli, PA). Neural decompression was performed based on the fracture pattern or neurologic symptoms. Patients with ligamentous injury involving the L5-S1 interval or had extensive posterior neural decompression involving the $\mathrm{S} 1$ facets and lamina underwent an associated L5-S1 posterolateral arthrodesis. Approaches were closed over drains and in anatomical layers. Skin was closed with vertical Allgower-Donati 3.0 nylon (Ethibond, Johnson \& Johnson, Norderstedt, Germany) sutures.

To compare groups, demographics were assessed, operative time, fluoroscopic time, radiation, and complications were delineated. Screw misplacement was assumed when screws were revised. Deep infection was defined as an infection requiring operative excisional debridement and antibiotic administration.

Data were analyzed using PASW® 18 (IBM, Armonk, NY). Descriptive statistics were completed. Chi-square and $t$ tests were used to compare those that had navigation aid versus those that did not, based on demographic data, potential contributing factors, OR time, fluoroscopy time, and radiation.

\section{Results}

Sixty-three (63) patients were included with a mean age of 58 years (range 18-87). There were 27/63 (43\%) males and $36 / 63(57 \%)$ females with a BMI of $26.2 \pm 5.6 \mathrm{~kg} / \mathrm{m}^{2}$. Length of hospital stay was averaged $33 \pm 26.2$ days. Reasons for instabilities and surgeries are listed in Table 1. Using the OTA/AO classification, all fractures were classified as 61 type $\mathrm{C}$ fractures.

\section{Groups}

During the study period, 32 patients underwent bilateral lumbopelvic fixation utilizing conventional fluoroscopic imaging alone (standard group) and 31 patients underwent the procedure with $3 \mathrm{D}$ navigated iliac screw placement (navigation group). No differences were found between the two groups regarding age $(59.9 \pm 18.4$ vs. $56.6 \pm 18.7$ years; $p=0.49)$, BMI $(26.0 \pm 6.0$ vs. $26.3 \pm 5.1 \mathrm{~kg} / \mathrm{m} 2 ; p=0.81)$, gender ( $59 \%$ vs. $58 \%$ females; $p=0.59)$, length of hospital stay $(35.2 \pm 24.7$ vs. $30.5 \pm 27.9$ days; $p=0.49)$, or comorbidities (Table 2).

Comparing screw length and diameter of the iliac screws, screw length averaged $111.6 \pm 7.7 \mathrm{~mm}$ in the standard group compared to $108.4 \pm 8.2 \mathrm{~mm}$ in the navigated group with a median of $110 \mathrm{~mm}$ in both groups 


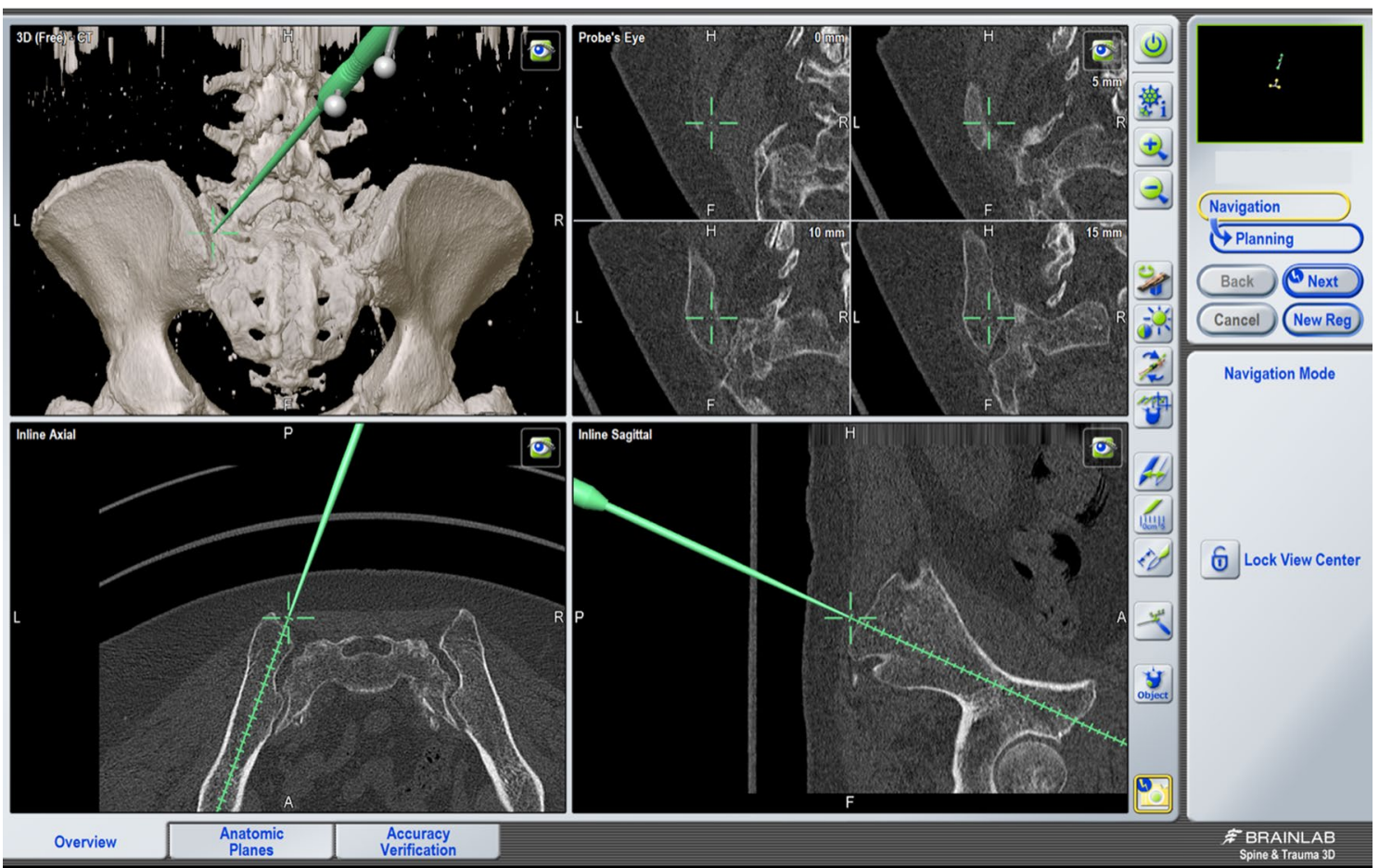

Fig. 4 Intraoperative view of the 3D navigation for iliac screw placement

Table 1 Lumbopelvic fixation was performed for different instability reasons

\begin{tabular}{llll}
\hline $\begin{array}{l}\text { Reason for } \\
\text { surgery }\end{array}$ & Standard group & Navigation group & Significance \\
\hline $\begin{array}{l}\text { Nonunion or } \\
\text { insufficient pre- } \\
\text { vious fixation }\end{array}$ & $5(16 \%)$ & $9(29 \%)$ & $p=0.12$ \\
$\begin{array}{l}\text { Spondylodiscitis } \\
\text { Tumor }\end{array}$ & $7(22 \%)$ & $2(6 \%)$ & $p=0.08$ \\
Fracture & $1(3 \%)$ & $0(0 \%)$ & $p=0.32$ \\
\hline
\end{tabular}

$(p=0.12)$. Screw diameter averaged $7.9 \pm 0.47 \mathrm{~mm}$ with a median of $8 \mathrm{~mm}$ in the standard group. All navigated screws were $8 \mathrm{~mm}$ in diameter $(p=0.40)$. Regarding additional procedures, in the standard group 15 patients (47\%) underwent additional decompression compared to 12 patients $(39 \%)$ in the navigated group $(p=0.51)$. The number of inserted pedicle screws averaged 2.92 in the standard group (median 2) with $66 \%$ at the L4 and/or L5 level and 3.0 in the navigated group (median 2$)(p=0.88)$.

\section{Surgical time}

Surgical time varied from 95 to 356 min with an average of $203 \mathrm{~min}$. Comparing both groups, OR time averaged $227.4 \pm 59.0 \mathrm{~min}$ in the standard group. In the navigated group, OR times was $176.7 \pm 64.9 \min (p=0.002)$ (Fig. 5).
Table 2 Comparison of demographics between standard and navigations groups

\begin{tabular}{llll}
\hline & Standard group & Navigation group & Significance \\
\hline Age (years) & 59.9 (SD 18.4) & 56.6 (SD 18.7) & $p=0.49$ \\
BMI $\left(\mathrm{kg} / \mathrm{m}^{2}\right)$ & $26.0($ SD 6.0) & 26.3 (SD 5.1) & $p=0.81$ \\
Females (\%) & 59 & 58 & $p=0.59$ \\
Length of hospital stay (days) & $35.2($ SD 24.7) & 30.5 (SD 27.9) & $p=0.49$ \\
Osteoporosis (\%) & 25 & 39 & $p=0.24$ \\
Cardiovascular disease (\%) & 53 & 39 & $p=0.26$ \\
Diabetes (\%) & 9 & 13 & $p=0.66$ \\
\hline
\end{tabular}

$S D=$ Standard Deviation 


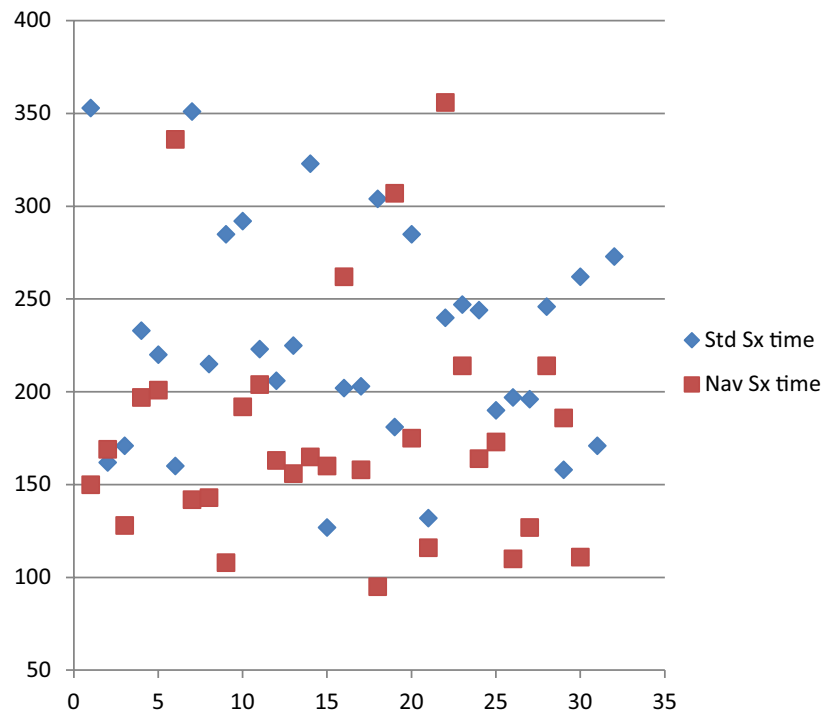

Fig. 5 Distribution of surgery time (minutes) for standard and navigated fixation

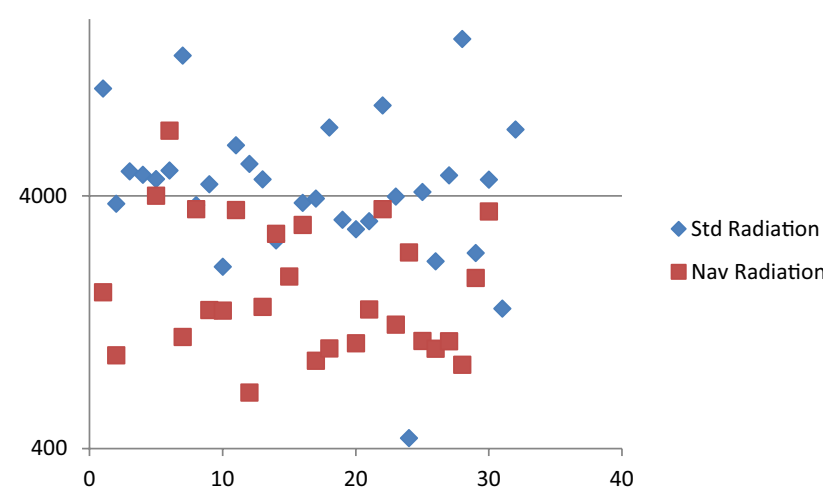

Fig. 6 Distribution of radiation $\left(\mathrm{Gy}^{*} \mathrm{~cm}^{2}\right)$ for standard and navigated fixation

\section{Fluoroscopy time}

Fluoroscopy time was $6.02 \pm 5.98 \mathrm{~min}$ for all patients combined. During standard lumbopelvic fixation an average of $8.6 \pm 7.2 \mathrm{~min}$ of fluoroscopy was utilized compared to $3.2 \pm 2.0 \mathrm{~min}$ in navigated cases $(p<0.001)$.

\section{Radiation exposure}

Patients and OR personnel were exposed to $3696.77 \pm 3142.86 \mathrm{~Gy}^{*} \mathrm{~cm}^{2}$ during lumbopelvic fixations. Average radiation exposure during surgery was reduced by navigation from $5130.8 \pm 3496 \mathrm{~Gy}^{*} \mathrm{~cm}^{2}$ to $2004.5 \pm 1465.2 \mathrm{~Gy}^{*} \mathrm{~cm}^{2}(p<0.001)$ (Fig. 6).

\section{Complications and accuracy of screw placement}

Additional surgeries were required in twenty-six patients (41.3\%). The reason for revision surgery was irrigation and debridement for hematoma or infection in 15 cases (23.8\%). Comparing both groups, additional surgeries were performed in 17 patients (53\%) in the standard group and nine patients $(30 \%)$ in the navigated group $(p=0.07)$. Infection was reduced in the navigated group compared to the standard group ( $7 \%$ vs. $28 \%$, respectively; $p=0.03$ ). The incidence of infection was not related to the OR time (201 min vs. $204 \mathrm{~min}$ ). One of the 63 patients had a malpositioned pedicle screw that needed revision (1.6\%). The malpositioned screw happened to be in the standard group (3\% vs. $0 \%$, respectively) but that was not significant $(p=0.33)$. Other reasons for revision surgeries were additional bone grafting for delayed spondylodesis (4), a disconnected drainage (1), extended decompression for persistent neurologic symptoms (1), and three patients required hardware exchange for broken rods in nonunion formation. Surprisingly, the iliac screws were still well-fixed and were not replaced during revision surgery. No iatrogenic neural injuries were recorded. One patient returned for hardware removal 1 year after the initial surgery.

\section{Discussion}

The key requirement of successful posterior pelvic ring repair is a proper alignment among the ilium, sacrum, and lumbar spine [15] that is stable enough to counterbalance translational and rotational forces in vertical and horizontal directions [6]. Lumbopelvic fixation transfers vertical loads from the ilium directly to the lumbar spine and prevents flexion of the pelvis [16]. The surgical technique is demanding with numerous potential complications [17]. In appropriately selected patients, high success rates can be achieved when it is performed systematically $[2,18]$. Biomechanical studies have confirmed that segmental lumbopelvic stabilization provides stable fixation of the posterior pelvic ring while unloading the area of injury $[6,19]$. Pedicle screw placement usually poses no difficulty and is performed routinely in most orthopedic clinics. One of the keystones for success of the lumbopelvic technique is the insertion of the iliac screws. Reliable screw positioning can be achieved utilizing multiple fluoroscopic views $[9,14]$. In the current study, only one malpositioned screw was observed in the standard group but that was no significant difference compared to the navigated group.

Standard screw placement may result in long radiation exposure, which is already increased for spinal surgeons and orthopedic OR staff [20-22]. Previous studies did show that navigation offers the possibility to reduce 
radiation exposure [23]. The current study shows that fluoroscopy time could be reduced by more than $60 \%$ from an average of $8.6 \pm 7.2$ min during standard lumbopelvic fixation to $3.2 \pm 2.0 \mathrm{~min}$ in procedures with navigated screw placement. This resulted in a radiation reduction of more than $50 \%$ by reducing the average radiation exposure during surgery by navigation from $5131 \mathrm{~Gy}^{*} \mathrm{~cm}^{2}$ to $2005 \mathrm{~Gy}^{*} \mathrm{~cm}^{2}$. In addition, the utilization of the surface matching combined with $3 \mathrm{D}$ navigation did not require additional CT scans. The necessary data were retrieved from the routinely performed CT scan that provided preoperative information on the extent of the injury.

Increased muscle mobilization and potential devitalization may increase the risk for deep hematoma formation and wound healing problems $[17,24]$. In previous studies, $26 \%$ of the patients required early secondary surgical procedures for wound-related complications [7]. In the current study, soft tissue-related complications were encountered in $24 \%$. Utilizing a more submuscular or minimal invasive approach to the PSIS may reduce soft tissue complications [25]. In our study, navigation did result in a reduced infection rate in the navigated group (7\%) compared to the standard group $(28 \%)(p=0.03)$. The reason remains unclear and no differences were documented in our medical charts. Localizing the entry point for the iliac screw with the pointer of the navigation system and a direct more submuscular approach may reduce soft tissue damage. Further studies determining factors that reduce wound healing problems are warranted.

We must admit limitations of our study. The major limitation of this study was its retrospective design. Therefore, no power analysis was conducted previously to assess sample sizes. This patient series represents complex instabilities all undergoing lumbopelvic fixation. All instabilities were complex and problematic being not amenable to traditional posterior pelvic ring fixation options. As a result, we also must acknowledge that a reasonable comparison of radiographic or clinical outcomes in a variety of instabilities was not possible.

The strength of this study is the number of patients included. To our knowledge, this study is the largest series comparing standard screw placement with navigated screw positioning in lumbopelvic fixations. All procedures were performed by trauma fellowship-trained orthopedic surgeons who have consistent treatment philosophies in one high volume level I trauma center. Navigated procedures were performed by the first author while the remaining surgeons continued standard screw placement. The initiation of the navigated procedure and the learning curve is included in the study. Therefore, this study has the potential to reflect realistic differences in OR time and fluoroscopy.

\section{Conclusion}

Fixation of sacral fractures continues to be challenging due to complex local anatomy. Especially in severe comminuted sacral fractures lumbopelvic fixation provides superior stability and allows immediate weight-bearing. For iliac screws, identifying the correct entry point and angle of implantation in all planes requires detailed anatomic knowledge and multiple radiographic views. For lumbopelvic fixation, 3D navigation may help to reduce operative time and fluoroscopy time resulting in a significant reduction of radiation exposure for the patient and OR personnel following the principle to minimize occupational radiation exposure "as low as reasonably achievable" [22].

Author contributions MH performed all data collection, interpretation, and writing the paper. MH contributed to study concept/design, data analysis, and interpretation. EY and DN helped with data analysis and interpretation. TS helped with interpretation and contributed to review of the final manuscript and final submission of the paper.

Funding Open Access funding enabled and organized by Projekt DEAL.

Availability of data and materials The datasets used and/or analyzed during the current study are available from the corresponding author on reasonable request.

\section{Compliance with ethical standards}

Conflict of interest The authors declare that they have no conflict of interests.

Ethics approval and consent to participate All investigations were conducted in conformity with ethical principles of research, and that informed consent for participation in the study was obtained. The Ethics Committee of the Ruhr-University Bochum approved the study protocol. The committee's reference number was 16-5711.

Open Access This article is licensed under a Creative Commons Attribution 4.0 International License, which permits use, sharing, adaptation, distribution and reproduction in any medium or format, as long as you give appropriate credit to the original author(s) and the source, provide a link to the Creative Commons licence, and indicate if changes were made. The images or other third party material in this article are included in the article's Creative Commons licence, unless indicated otherwise in a credit line to the material. If material is not included in the article's Creative Commons licence and your intended use is not permitted by statutory regulation or exceeds the permitted use, you will need to obtain permission directly from the copyright holder. To view a copy of this licence, visit http://creativecommons.org/licenses/by/4.0/.

\section{References}

1. Tile M (1984) Fractures of the pelvis and acetabulum. Lippincott Williams \& Wilkins, Baltimore 
2. Moshirfar A, Rand FF, Sponseller PD et al (2005) Pelvic fixation in spine surgery Historical overview indications biomechanical relevance and current techniques. J Bone Jt Surg Am 87(Suppl 2):89-106

3. Griffin DR, Starr AJ, Reinert CM et al (2003) Vertically unstable pelvic fractures fixed with percutaneous iliosacral screws: Does posterior injury pattern predict fixation failure? J Orthop Trauma 17:399-405

4. Kaech K, Trenz O (1994) Distraction spondylodesis of the sacrum in "vertical shear lesions" of the pelvis. Unfallchirurg 97:28-38

5. Barcellos ALL, da Rocha VM, Guimaraes JAM (2017) Current concepts in spondylopelvic dissociation. Injury 48(Suppl 6):S5-S11

6. Schildhauer TA, Josten C, Muhr G (1998) Triangular osteosynthesis of vertically unstable sacrum fractures: a new concept allowing early weight-bearing. J Orthop Trauma 12:307-314

7. Schildhauer TA, Bellabarba C, Nork SE et al (2006) Decompression and lumbopelvic fixation for sacral fracture-dislocations with spino-pelvic dissociation. J Orthop Trauma 20:447-457

8. Marintschev I, Gras F, Klos K et al (2010) Navigation of vertebro-pelvic fixations based on CT-fluoro matching. Eur Spine J 19:1921-1927

9. Gothner M, Dudda M, Schildhauer TA (2013) Anatomical and radiological aspects in lumbopelvic fixation. Unfallchirurg 116:979-984

10. Grossterlinden L, Rueger J, Catala-Lehnen P et al (2011) Factors influencing the accuracy of iliosacral screw placement in trauma patients. Int Orthop 35:1391-1396

11. Zwingmann J, Konrad G, Kotter E et al (2009) Computer-navigated iliosacral screw insertion reduces malposition rate and radiation exposure. Clin Orthop Relat Res 467:1833-1838

12. Garrido BJ, Wood KE (2011) Navigated placement of iliac bolts: description of a new technique. Spine J 11:331-335

13. Gruen GS, Leit ME, Gruen RJ et al (1995) Functional outcome of patients with unstable pelvic ring fractures stabilized with open reduction and internal fixation. J Trauma 39:838-844 (discussion 44-5)

14. Schildhauer TA, McCulloch P, Chapman JR et al (2002) Anatomic and radiographic considerations for placement of transiliac screws in lumbopelvic fixations. J Spinal Disord Tech 15:199-205 (discussion)
15. Henderson RC (1989) The long-term results of nonoperatively treated major pelvic disruptions. J Orthop Trauma 3:41-47

16. Schildhauer TA, Ledoux WR, Chapman JR et al (2003) Triangular osteosynthesis and iliosacral screw fixation for unstable sacral fractures: a cadaveric and biomechanical evaluation under cyclic loads. J Orthop Trauma 17:22-31

17. Sagi HC, Militano U, Caron T et al (2009) (2009) A comprehensive analysis with minimum 1-year follow-up of vertically unstable transforaminal sacral fractures treated with triangular osteosynthesis. J Orthop Trauma 23:313-319 (discussion 9-21)

18. Jones CB, Sietsema DL, Hoffmann MF (2012) Can lumbopelvic fixation salvage unstable complex sacral fractures? Clin Orthop Relat Res 470:2132-2141

19. Keel MJ, Benneker LM, Siebenrock KA et al (2011) Less invasive lumbopelvic stabilization of posterior pelvic ring instability: technique and preliminary results. J Trauma 71:E62-E70

20. Rampersaud YR, Foley KT, Shen AC et al (2000) Radiation exposure to the spine surgeon during fluoroscopically assisted pedicle screw insertion. Spine 25:2637-2645

21. Theocharopoulos N, Perisinakis K, Damilakis J et al (2003) Occupational exposure from common fluoroscopic projections used in orthopaedic surgery. J Bone Jt Surg Am 85:1698-1703

22. Hayda RA, Hsu RY, DePasse JM et al (2018) Radiation exposure and health risks for orthopaedic surgeons. J Am Acad Orthop Surg 26:268-277

23. Kim CW, Lee YP, Taylor W et al (2008) Use of navigationassisted fluoroscopy to decrease radiation exposure during minimally invasive spine surgery. Spine J 8:584-590

24. Bellabarba C, Schildhauer TA, Vaccaro AR et al (2006) Complications associated with surgical stabilization of high-grade sacral fracture dislocations with spino-pelvic instability. Spine 31:S80 S88 (discussion S104)

25. Williams SK, Quinnan SM (2016) Percutaneous lumbopelvic fixation for reduction and stabilization of sacral fractures with spinopelvic dissociation patterns. J Orthop Trauma 30:e318-e324

Publisher's Note Springer Nature remains neutral with regard to jurisdictional claims in published maps and institutional affiliations. 